\title{
Sub-Millisecond Transient Analysis with Multi-Point Measurement in Weak Grids
}

\author{
A. Matthee \\ University of Twente \\ Enschede, The Netherlands \\ a.matthee@utwente.nl
}

\author{
N. Moonen \\ University of Twente \\ Enschede, The Netherlands
}

\author{
F. Leferink \\ University of Twente, Enschede \\ Thales, Hengelo \\ The Netherlands
}

\begin{abstract}
Peak loads determine the rated capacity of islanded grid power supplies. This often results in non-optimal running conditions in terms of cost, efficiency or size footprint. Presented in this paper is a method of in-depth analysis of very fast submillisecond transient behaviour performed between sub-systems and supply in a low-inertia grids. Using this method, time domain measurements can be analysed to potentially achieve electromagnetic compatibility. Synchronised current and voltage measurements are possible, with offsets in the sub-milliseconds and high sample rates of tens of Mega-samples per second. A demonstration of a weak grid is presented showing effects of linear and non-linear loads on the supply source and power quality.
\end{abstract}

Index Terms-Power quality, Generator efficiency, Raspberry Pi, Multi-point measurement, Electromagnetic interference, Smart Grids

\section{INTRODUCTION}

Recently, electromagnetic compatibility (EMC) challenges of low inertia grids has come to the fore due to rapid implementation and increased exposure to the latest power electronics innovations [1]-[3], amongst other reasons. Electromagnetic interference (EMI) cases are reported due to the absence of a "high-inertia" distribution network. This causes rapid voltage changes (RVC) in the power supply, resulting in conducted EMI issues [4]-[6]. A well known concern in islanded or low inertia grids is the requirement to over-specify the electrical supply source to compensate for EMI, due to for instance voltage sags, caused by transients or cold start condition and inrush currents [7]-[9]. An example of this would be a vehicle carrying a complex microgrid system as a payload with a generator capacity of $40 \mathrm{~kW}$ [10]. Although the average load measured in this case is roughly $23 \mathrm{~kW}$ during full system operation. With further investigation the generator capacity can potentially be reduced. This has huge cost, weight and volume consequences. Increased cost and reliability are massive inhibitors of the adoption of renewable energy sources, however in case of fossil fuel powered generators the effect is similar regarding the optimal operating conditions, initial capacity determination and reliability. Diagnosis of microgrid transient instability issues requires insights into behaviour of sub-systems.

Etopia project research is funded by the European Union's Horizon 2020 research and innovation programme under the Marie Sklodowska-Curie grant agreement No 812753
In [11] an analysis method has been presented to determine the impulsiveness of a load in a system. Next to stochastic and cyclo-stationary noise sources, highly transient effects are introduced into systems through the use of (non-linear) loads switching between operational modes, i.e. very complex systems containing events that do not have a stochastic nature but are still devastating in case of optimal efficiency design. Monitoring systems of transients and grid stability do exist such as [12] or [13] as well as products such as the PQube [14]. Another widely known method is synchrophasors or phasor measurement unit (PMU) [15]-[17] for large grid stability or power flow analysis and fault diagnosis. In large complex systems the purpose is finding the source of major inrush current and propagation of RVC. Renewable sources in particular due to the inherent intermittency of the supply [8], [18] adding another layer of complexity. In this paper a system is proposed which performs synchronisation of measurement data in the MS/s range between multiple sub-system nodes within submillisecond precision. Very fast, short time-frame transients can accurately be determined improving the ability to perform design decisions for EMC and more accurate source capacity determination. Thus improving overall system effectiveness per unit cost-, footprint volume-and-performance. This system is composed of multiple components interfaced with software to seamlessly provide synchronised measurements of transient events with high sample rates. Versatility in configuration with a relatively small footprint-and-cost coupled with plug and play properties are inherent in this proposed system. With this stated practical implementation of the concept is the principle target and thus applied to all aspects developed as far as possible.

In section III a demonstration of the proposed system is evaluated using an emulation of a weak grid. The test environment is set up as a simplistic example of a use case. The setup is arranged with a measurement point placed on the output of a four quadrant amplifier as the weak supply. Two more measurement points which measure sub-branches, one being a purely linear load and another a non-linear load consisting of a light emitting diode (LED) light bank and dimmer circuit connected to a water pump, both known for high harmonic current distortion [3], [19], [20]. Current and voltage waveforms are measured on the three nodes and can be analysed accurately. The specific demonstrator discussed 
in section III consists of three measurement nodes but it is important to note a complex system would consist of many more, the proposed system is scalable to what is practically achievable via a local network configuration and processing capabilities of the master node (MN). The results of the demonstrator are presented and discussed in section IV.

\section{System OVERVIEW}

The system described in this paper consists of multiple components, as shown in Fig. 1. Interface between devices is achieved via Universal Serial Bus (USB) and Ethernet. The MN may consist of a personal computer (PC). The $\mathrm{MN}$ is connected to multiple slave node ( $\mathrm{SN}$ ) devices via Ethernet and a network switch, local area network (LAN) or even WIFI. The SN is a Raspbery Pi 4 (RP4) device and processes data from a Picoscope 5444D MSO (high sample rate oscilloscope) connected via USB. The Picoscope measures the voltage and current by means of differential voltage probes and current clamps. The $\mathrm{SN}$ is placed in any point of interest to measure voltage and current waveforms. Multiple devices can be utilised simultaneously as each operates independently with the exception of the commands sent from the MN. Limitations of random access memory (RAM) or storage memory of the MN or SN may constrain sample frequency and sample time, this should be taken into consideration before implementation. Usually in the form of a trade-off between sample frequency and sample duration.

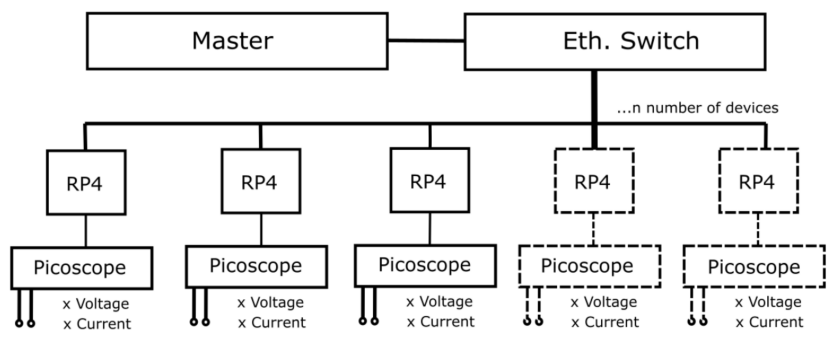

Fig. 1: System overview diagram

Each Picoscope is configured with a unique channel setup, depending on the requirements. The data is streamed from the Picoscope via USB to the RP4. Data is processed accordingly either as a sub-process on the $\mathrm{SN}$ due to its multi processing core functionality or post-measurement combined datasets on the MN. In case of massive data sets, data may be moved to more powerful computational devices for analysis. Measurement data is combined with a master slave approach over ethernet. The master device consist of a personal computer, in this case a laptop connected to multiple Raspberry Pi slave devices via a network switch. The Picoscope is capable of up to $80 \mathrm{MS} / \mathrm{s}$ measurement of voltage and current waveforms. However the data processing and storage capability of the RP4 limits this sample period of the picoscope.

Depending on the requirements of the system under test (SUT) a trade-off must be made in order to satisfy the performance criteria of the slave devices and system overall. The data is streamed to the SN from the Picoscope and stored temporarily on the SN. Once the data streaming is completed the data is retrieved by the $\mathrm{MN}$ via Ethernet and combined and processed. It is now possible to analyse data from multiple nodes measured in the sub-system time synchronised to within $1 \mathrm{~ms}$. It is apparent a major factor in the time offset is contributed by the speed of the ethernet switch. This should be considered as a factor in the importance of accuracy required for a specific use case.

The synchronisation between devices should have a maximum time offset of less than half of the fundamental frequency measured to align relative half cycles in post processing with certainty. A smaller time offset is of course desirable. Should it be required, data may also be streamed continuously via ethernet. Each dataset contains the trigger time stamp, an important point to note is that an external trigger, driven by the RP4, with precise time stamp trigger fire is required to compensate for any Picoscope triggering signal time deviation. The delay in data acquisition can be in the tens of milliseconds from initiation of streaming commands thus external triggering is required. The trigger mechanism consists of a pulse generated by a GPIO pin on the RP4 connected to a channel on the Picoscope. The pulse is fired at a precise time stipulated by the $\mathrm{MN}$ and provides a reference point between all SN. The Picoscope detects the trigger on the rising edge of the pulse. During data acquisition the trigger signal index is marked in the data which is used to synchronise all measured waveforms. To synchronise the internal clock of the RP4, which is known to drift, a time-stamp is sent from the $\mathrm{MN}$ as an argument which forms part of the start command. All SN there have internal clocks synchronised to the master and the trigger event is set to commence at a predetermined time system wide. This short synchronisation offset allows waveforms to be compared directly by a voltage cycle in any other measurement point in the system. The data can therefore be shifted in the post-processing according to the trigger index, and if required for more precision, phase to align zero crossings for any slight deviation between datasets. Post measurement the data is copied to the MN via SSH commands and processed. The Picoscope channel data used for external trigger is analysed in order to obtain the synchronisation point offset for each SN.

\section{Measurement SETUP}

In Fig. 2 a diagram of the test setup is shown which follows the same layout as described in Fig. 1, although the measurement points are limited to three in this case, labelled 1,2 and 3. The load connected to branch (2) and (3) is listed in Table. I.A Pacific Power Smart Source 140-TMX AC power supply, which is a 4-quadrant amplifier, acts as the weak or low inertia source. Differential voltage probes and current clamps measure voltage and current and are connected to the relevant Picoscopes for each SN. The system measures voltage and current at all three branches simultaneously, comparing the results will demonstrate the operation of the system. All SNs are connected via LAN communicate with the master as described in section II. Theoretically these devices can be 
placed in completely separate areas even long distances apart with minimal effect on the performance.

TABLE I: Branch loads

\begin{tabular}{|c|c|c|}
\hline Branch Number & Description & Nominal Power (W) \\
\hline$(2)$ & Resistor bank & 900 \\
$(3)$ & LED light bank (32x2W) & 64 \\
$(3)$ & Water pump with dimmer & 40 \\
\hline
\end{tabular}

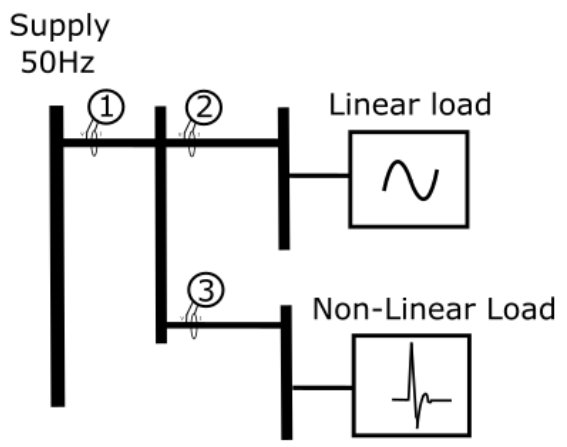

Fig. 2: Single line diagram of system demonstrator and measurement points 1,2 and 3

As illustrated in Fig. 2, on branch (2), a linear load has been connected consisting of a resistor bank. On branch (3), a non-linear load is connected consisting of a LED light bank and dimmer controlled pump known for significant transient current draws. Branch (2) and (3) is supplied power by branch (1). The purpose of the system is to measure the extent of EMI in real world applications. The aim of this demonstration is to provide a lab controlled validation setup for diagnosis of a system with a disturbance causing load in an unknown subsystem. It is expected that the linear load will draw sinusoidal current with minimal distortion of the supply while the nonlinear load should cause significant transients and stress on the supply.

\section{Measurement Results}

The test procedure includes starting the voltage supply (four quadrant amplifier) at zero load. This four quadrant amplifier emulates a renewable energy source or a local small generator. Once the measurement devices are activated and triggered the load in branch (2) is activated. As seen in Fig. 3 the current drawn from this load is linear and with a constant sinusoidal amplitude. Also indicated in Fig. 3, shortly afterwards the non-linear load or branch (3) is energised. The energise time offset between branches is to clearly show the difference in response between sub-systems. This results in inrush current pulse, which exceeds $17 \mathrm{~A}$ caused by capacitors in the power electronics shown at approximately $1653 \mathrm{~ms}$ on the sampling time window scale in Fig. 4. Shortly after, near the peak of the next half cycle at $1660 \mathrm{~ms}$, another larger current pulse is present and repeated in the next negative half-cycle. Likely caused by current draw in the power electronics circuitry to enable operation of the systems. The peak of this transient current pulse is approximately $31 \mathrm{~A}$. After a settling period whereby the LED bank luminaires activate and the pump begins operation more current pulses are apparent but not in regular amplitudes nor precisely periodic.

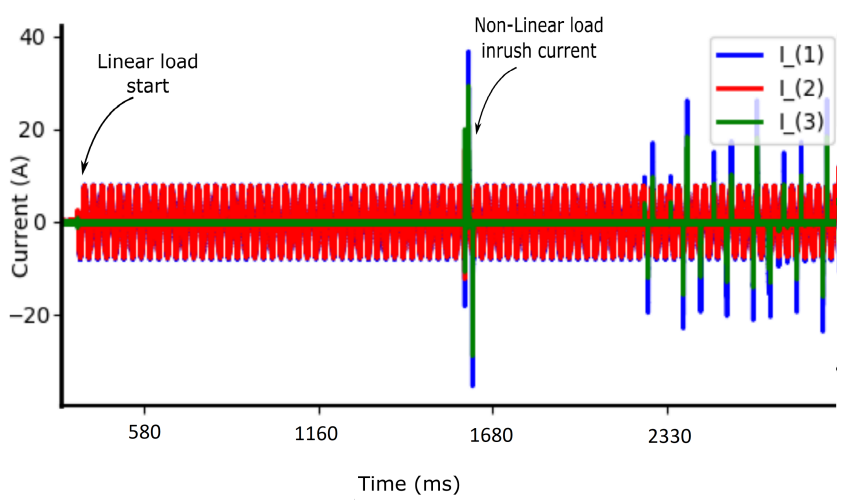

Fig. 3: Current profile vs measurement window time for branch (1), (2) and (3)

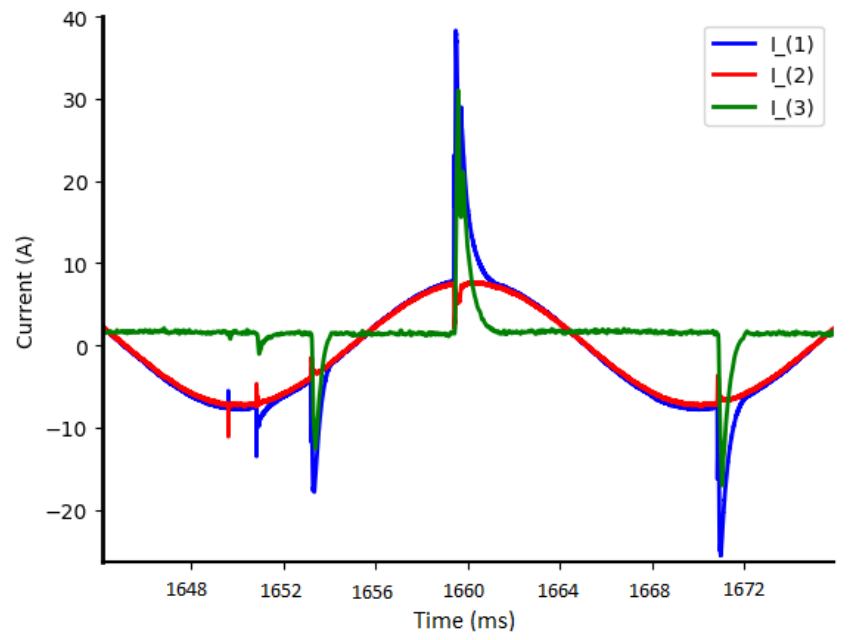

Fig. 4: Sub cycle current vs time in branch (1), (2) and (3)

These two branch currents sum to the total draw of the supply of course. Therefore the current measured at branch (1) with inrush and secondary peak of (3) is $17.8 \mathrm{~A}$ and 38.3 A respectively. In Table. II the root mean square (RMS) currents and power, peak current and inrush current is shown as measured in each branch. Also, a ratio $\left(\right.$ Ratio $\left._{I}\right)$, is shown of the peak measured current in each branch compared to the RMS current per branch.

TABLE II: Current and power values per branch

\begin{tabular}{|c|c|c|c|c|c|}
\hline No. & $I_{R M S}(\mathrm{~A})$ & $I_{p k}(\mathrm{~A})$ & $P_{R M S}(\mathrm{~W})$ & $I_{\text {inrush }}(\mathrm{A})$ & Ratio $_{I}$ \\
\hline$(1)$ & 5.65 & 38.3 & 846 & 17.8 & 6.7 \\
$(2)$ & 4.89 & 11.2 & 713 & 11.2 & 2.3 \\
$(3)$ & 0.39 & 31 & 58 & 12.5 & 78 \\
\hline
\end{tabular}

Should a more complex system, containing many such transient current pulses, be present the behaviour and reliability of the supply would be further challenged. The voltage deviation 
caused by the transient current pulse is shown in Fig. 5. A closer look at the effects on the supply and system voltage at various points is shown in Fig. 6. The plot is shown of the positive half cycle deviation of measurement time between $1658.4 \mathrm{~ms}$ to $1660.8 \mathrm{~ms}$ at measurement points 1,2 and 3 . It must be mentioned that the voltage plots has small time offsets due to non-perfect triggering between SNs.

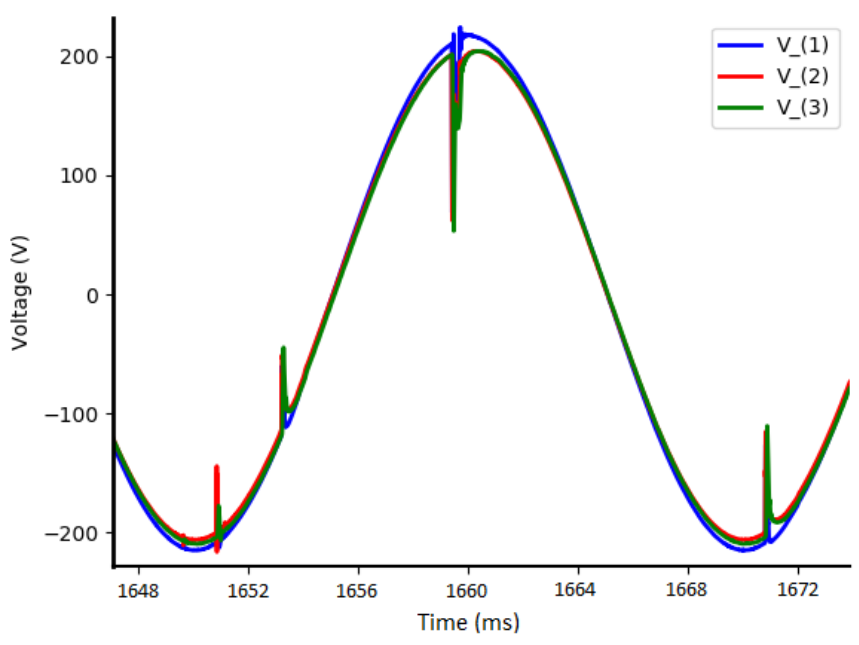

Fig. 5: Inrush period voltage vs time - branch (1), (2), (3)

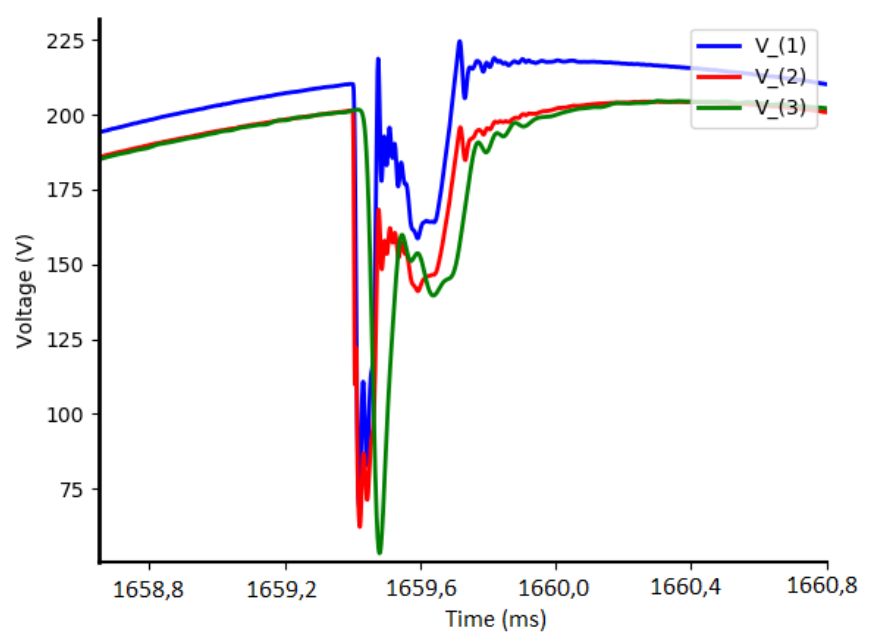

Fig. 6: Sub-millisecond range inrush voltage deviation

The voltage dip seen by all branches reaches as low as $54 \mathrm{~V}$ from a voltage $187 \mathrm{~V}$ at that specific instantaneous moment in the voltage cycle and last for $0.344 \mathrm{~ms}$ in branch (3). The equates to a voltage draw down of over $71 \%$. This falls below the $20 \%$ limit of STANAG 1008 standard. The voltage recovery in branch (2) takes a longer due to the resistive and inductive components of the load. Due to the disturbance in branch (3) the voltage and current of branch (2) are also effected. In this specific case the operation of the load in branch (2) is unaffected but for more sensitive loads a high probability of malfunction exists. Also, the significant stress placed on the supply could cause disruptions or overloads resulting in total system shutdown. The significant voltage dip in the system can be explained by the low inertia or weak nature of the source. In case of a strong grid the deviations in voltage may be less severe.

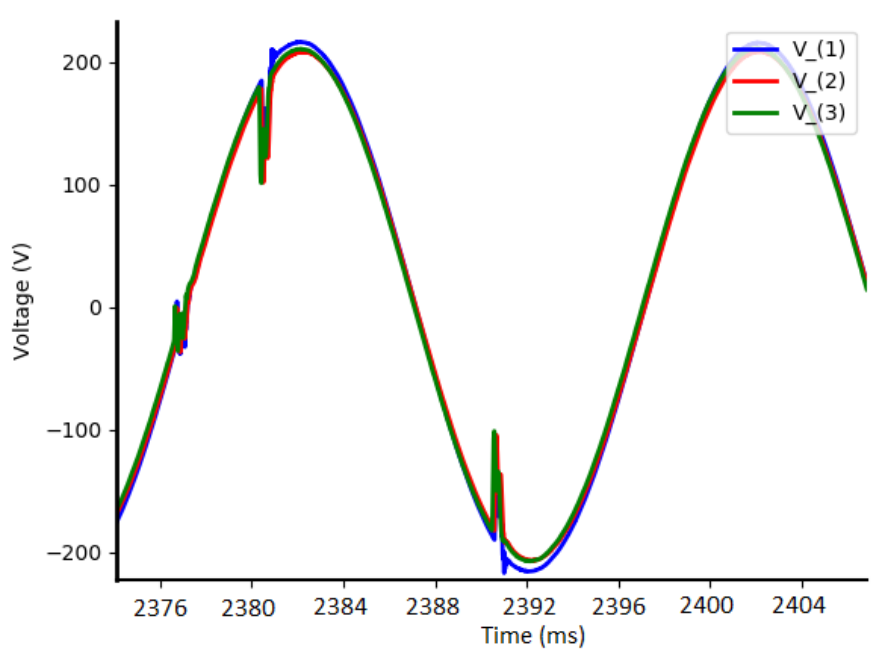

Fig. 7: Measured voltage in branch (1), (2) and (3) with additional capacitor on (3)

A common engineering practice would include adding a capacitor to the system in order to smooth out current transients. To observe the difference in system behaviour a $17.7 \mu \mathrm{F}$ capacitor is added to branch (3) as the known source of the large current pulses. Fig. 7 the voltage waveform is shown for all branches in the same inrush current phase as shown in Fig. 6. The inrush current in this case occurs near the zero crossing in the voltage cycle. As a results the inrush current, shown in Fig. 8, is significantly lower. Also the voltage dip on the all measurement points show a less severe draw down especially on the supply which measures a voltage drop from $184 \mathrm{~V}$ to $111 \mathrm{~V}$. In the case where the branch is energised on the peak of the voltage waveform and not on or near the zero crossing, the situation is much different. Although the additional capacitor decreased the effect of periodic current transients (after inrush current) on voltage deviation of the supply, the initial inrush current pulse is significantly increased.

The voltage deviation on all measured nodes is shown in Fig. 9. The voltage draw down to zero with very short time reversal on measurement point (1) most likely due to reactive currents flowing through the system in a phase of instability of the source. The inrush current is shown in Fig. 10. The peak of the pulse is measured at 54.9A in branch (1), a $44 \%$ increase over the previous highest maximum peak current. In Table. III the RMS currents and power, peak current and inrush current is shown for the measured changes after capacitor addition.

The peak currents measured on the power draw of the nonlinear load operational current is slightly increased to $32.8 \mathrm{~A}$. 


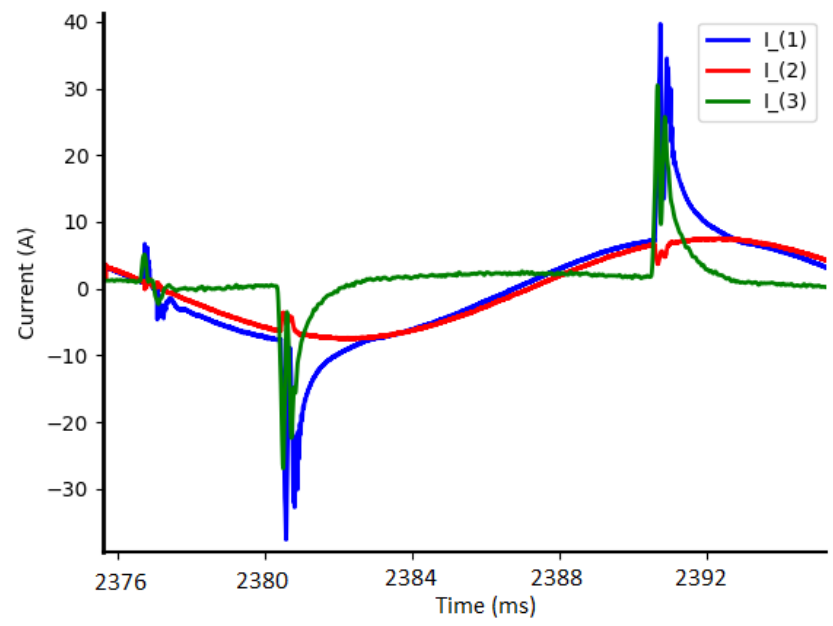

Fig. 8: Sub-cycle current vs time in branch (1), (2) and (3)

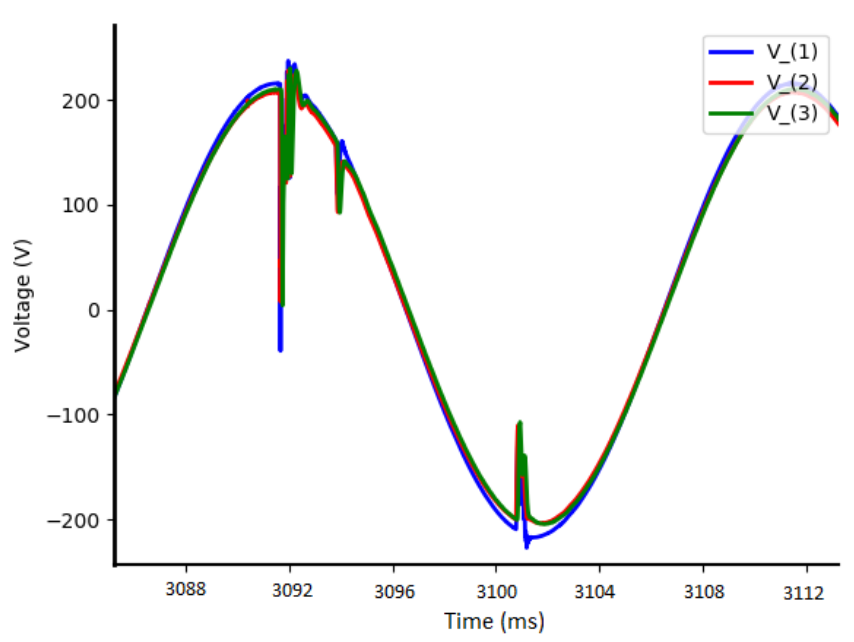

Fig. 9: voltage vs time in branch (1), (2) and (3) with inrush on peak of voltage cycle

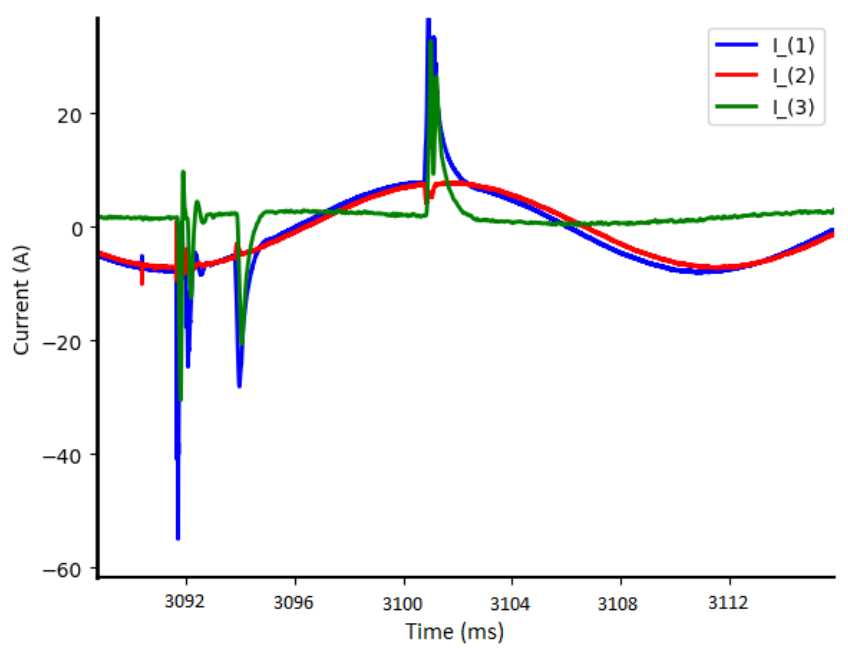

Fig. 10: Sub-cycle voltage vs time in branch (1), (2) and (3)
TABLE III: Current and power values per branch with added capacitor on branch (3)

\begin{tabular}{|c|c|c|c|c|c|}
\hline No. & $I_{R M S}(\mathrm{~A})$ & $I_{p k}(\mathrm{~A})$ & $P_{R M S}(\mathrm{~W})$ & $I_{\text {in-rush }}$ & Ratio \\
\hline$(1)$ & 5.75 & 54.9 & 876 & 54.9 & 9.6 \\
$(2)$ & 4.91 & 10.2 & 715 & 10.2 & 2.0 \\
$(3)$ & 0.49 & 32.8 & 72 & 32.8 & 475.1 \\
\hline
\end{tabular}

Hence adding a capacitor as current transient mitigation measure may not be the solution, especially in the case of large inrush currents interfering with system operation.

From this measurement validation setup, valuable information has been obtained on supply and sub-system power conditions. A real world application will next be approached to optimise power supply, improve power quality (PQ) and prevent EMI. In the future. models are to be developed to predict related issues and this measurement setup can be used for verification and validation of simulations.

\section{CONCLUSION}

Mobile systems operated by (diesel) generators, and weak or islanded micro-grids supplied by renewable energy sources, are conventionally over-designed to prevent conducted electromagnetic interference problems due to voltage sags and dips caused by inrush currents from, often non-linear, loads. The system proposed in this paper allows synchronized multichannel voltage and current measurements within a large complex installation. It offers assistance on the in depth analysis of such short-time frame transient events with near realtime synchronisation between measurement points of current and voltage of less than $1 \mathrm{~ms}$ possible. A simple test case is presented showing sub-system load behaviour where the system demonstrates its ability to identify the source and magnitude of volatile load transient events. The extent of the stress placed on a low inertia supply becomes apparent and sub-systems experiencing voltage dips of over $39 \%$ as well as current pulses of 5 times the average current of approximately 8 A. The developed system is being used for measurements in a demonstrator of a new complex system, and to support the proper choices of the energy supply of the series product. The system will mainly be used for validation of models to predict electromagnetic interference in the concept and design phase of a complex system.

\section{REFERENCES}

[1] Y. Liu, J. Peng, G. Wang, H. Wang, and K. Y. See, "LCL filter design and analysis of grid-connected converter for power quality and EMI compliance," 2016 Asia-Pacific International Symposium on Electromagnetic Compatibility, APEMC 2016, vol. 01, no. 4, pp. 142144, 2016.

[2] P. M. Ivry, D. W. P. Thomas, and M. Sumner, "Assessment of power quality in a microgrid with power electronic converters," 2016 Asia-Pacific International Symposium on Electromagnetic Compatibility, APEMC 2016, no. 1, pp. 825-827, 2016.

[3] R. B. Timens, F. J. K. Buesink, V. Ćuk, J. F. G. Cobben, W. L. Kling, and F. B. J. Leferink, "High harmonic distortion in a new building due to a multitude of electronic equipment," IEEE International Symposium on Electromagnetic Compatibility, pp. 393-398, 2011. 
[4] S. Arif and T. Aziz, "Study of Transient Stability with Battery Energy Storage Systems in Renewable Integrated Islanded Microgrid," WIECON-ECE 2017 - IEEE International WIE Conference on Electrical and Computer Engineering 2017, no. December, pp. 266-269, 2018.

[5] H. Hilal, M. A. Oktaufik, A. Prastawa, B. Prasetyo, and R. Hutahaean, "Smart Diesel Generator to compensate on-grid PV fluctuation: A case study in Sumba Island Indonesia," 3rd IEEE Conference on Power Engineering and Renewable Energy, ICPERE 2016, pp. 33-37, 2017.

[6] Y. Wang, T. Phung, and J. Ravishankar, "Impact of battery storage on micro-grid transient performance," IEEE Region 10 Annual International Conference, Proceedings/TENCON, vol. 2015-Janua, pp. 1-6, 2015.

[7] R. Singh and M. Kirar, "Transient stability analysis and improvement in microgrid," International Conference on Electrical Power and Energy Systems, ICEPES 2016, no. i, pp. 239-245, 2017.

[8] R. Belkacemi, S. Zarrabian, A. Babalola, and R. Craven, "Experimental Transient Stability Analysis of MicroGrid systems: Lessons learned," IEEE Power and Energy Society General Meeting, vol. 2015-Septe, pp. $1-5,2015$.

[9] O. Bassey, S. Member, K. L. Butler-purry, and B. Chen, "Active and Reactive Power Sharing in Inverter Based," no. 1, 2019.

[10] B. Have, N. Moonen, and F. Leferink, "On-Site Efficiency Analysis of a Generator in the Millisecond Range," EMC Europe 2020 - 2020 International Symposium on Electromagnetic Compatibility.

[11] T. Hartman, M. Pous, M. A. Azpúrua, F. Silva, and F. Leferink, "Onsite Waveform Characterization at Static Meters Loaded with Electrica Vehicle Chargers," EMC Europe 2019 - 2019 International Symposium on Electromagnetic Compatibility, pp. 191-196, 2019.

[12] G. Rietveld, J. P. Braun, R. Martin, P. Wright, W. Heins, N. Ell, P. Clarkson, and N. Zisky, "Measurement Infrastructure to Support the Reliable Operation of Smart Electrical Grids," IEEE Transactions on Instrumentation and Measurement, vol. 64, no. 6, pp. 1355-1363, 2015.
[13] Q. Xaunwei, L. Chengyu, W. Song, H. Liexiang, Q. Yutao, and S. Baoxing, "The Full Frequency-domain Transient Recorder and the Analysis System," 2019 IEEE PES Innovative Smart Grid Technologies Asia, ISGT 2019, no. 521104170012, pp. 1154-1159, 2019.

[14] A. M. Dumitrescu, R. Roman, and M. Albu, "Synchronized measurements and power quality assesment," 2015 IEEE Eindhoven PowerTech PowerTech 2015, pp. 2-7, 2015.

[15] M. H. Cintuglu, A. T. Elsayed, and O. A. Mohammed, "Microgrid automation assisted by synchrophasors," 2015 IEEE Power and Energy Society Innovative Smart Grid Technologies Conference, ISGT 2015, pp. $1-5,2015$.

[16] E. O. Schweitzer, A. Guzman, H. J. Altuve, D. A. Tziouvaras, and J. Needs, "Real-time synchrophasor applications in power system control and protection," IET Conference Publications, vol. 2010, no. $558 \mathrm{CP}$ 2010.

[17] G. C. Patil and A. G. Thosar, "Application of synchrophasor measurements using PMU for modern power systems monitoring and control," 6th International Conference on Computation of Power, Energy, Information and Communication, ICCPEIC 2017, vol. 2018-Janua, pp. 754-760, 2018

[18] I. Vokony and A. Dan, "Examination of smart grids in island operation," 2009 IEEE Bucharest PowerTech: Innovative Ideas Toward the Electrical Grid of the Future, pp. 1-7, 2009.

[19] G. Rata and M. Rata, "The study of harmonics from dimmable LED lamps, using CompactRIO," 2016 13th International Conference on Development and Application Systems, DAS 2016 - Conference Proceedings, pp. 180-183, 2016.

[20] B. T. Have, T. Hartman, N. Moonen, and F. Leferink, "Inclination of Fast Changing Currents Effect the Readings of Static Energy Meters," EMC Europe 2019 - 2019 International Symposium on Electromagnetic Compatibility, pp. 208-213, 2019. 